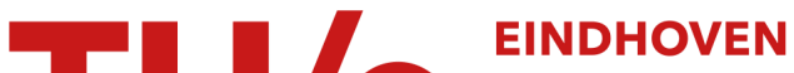 UNIVERSITY OF TECHNOLOGY
}

\section{Regular perturbation for the weak-dispersion regime}

\section{Citation for published version (APA):}

Oliari, V., Agrell, E., \& Alvarado, A. (2019). Regular perturbation for the weak-dispersion regime. In 21st International Conference on Transparent Optical Networks, ICTON 2019 [8840474] Institute of Electrical and Electronics Engineers. https://doi.org/10.1109/ICTON.2019.8840474

DOI:

10.1109/ICTON.2019.8840474

Document status and date:

Published: 19/09/2019

\section{Document Version:}

Accepted manuscript including changes made at the peer-review stage

\section{Please check the document version of this publication:}

- A submitted manuscript is the version of the article upon submission and before peer-review. There can be important differences between the submitted version and the official published version of record. People interested in the research are advised to contact the author for the final version of the publication, or visit the $\mathrm{DOI}$ to the publisher's website.

- The final author version and the galley proof are versions of the publication after peer review.

- The final published version features the final layout of the paper including the volume, issue and page numbers.

Link to publication

\section{General rights}

Copyright and moral rights for the publications made accessible in the public portal are retained by the authors and/or other copyright owners and it is a condition of accessing publications that users recognise and abide by the legal requirements associated with these rights.

- Users may download and print one copy of any publication from the public portal for the purpose of private study or research.

- You may not further distribute the material or use it for any profit-making activity or commercial gain

- You may freely distribute the URL identifying the publication in the public portal.

If the publication is distributed under the terms of Article 25fa of the Dutch Copyright Act, indicated by the "Taverne" license above, please follow below link for the End User Agreement:

www.tue.nl/taverne

Take down policy

If you believe that this document breaches copyright please contact us at:

openaccess@tue.nl

providing details and we will investigate your claim. 


\title{
Regular perturbation for the weak-dispersion regime
}

\author{
Vinícius Oliari $^{1 *}$, Erik Agrell $^{2}$, Alex Alvarado ${ }^{1}$ \\ ${ }^{1}$ Department of Electrical Engineering, Eindhoven University of Technology (TU/e), 5600MB Eindhoven, The Netherlands \\ ${ }^{2}$ Department of Electrical Engineering, Chalmers University of Technology, Gothenburg SE-41296, Sweden \\ ${ }^{*}$ E-mail: v.oliari.couto.dias@tue.nl
}

\begin{abstract}
We present a novel analytical model for the lossless fiber in the weak-dispersion regime. The model is compared to three other models via simulations using the split-step Fourier method. These simulations explore the validity of the models with respect to parameters that affect the signal after the fiber propagation. In one of the systems under consideration, the proposed model is accurate until $8.7 \mathrm{dBm}$ while the others are accurate only until 2.4 $\mathrm{dBm}$. The impact of the modulation format is analyzed for three of the models. Our results show differences up to $1.82 \mathrm{~dB}$ in the maximum power in which the proposed model remains accurate when changing from quadrature phase shifting keying to 8-ary quadrature amplitude modulation.
\end{abstract}

Keywords: High nonlinearity regime, nonlinear Schrödinger equation, optical fibers, Raman amplification, weakly dispersion regime.

\section{INTRODUCTION}

The transmission of a signal in an optical fiber is characterized by linear and nonlinear effects [1, Ch. 2]. The linear effects are related to attenuation and chromatic dispersion [1, Ch. 3]. The nonlinear effect can be modeled by the Kerr effect [1, Ch. 4]. The attenuation can be neglected when considering ideal Raman amplification, which maintains the signal power constant during propagation [2]. In that case, the only linear effect remaining is the chromatic dispersion, which can be easily compensated at the receiver by a linear filter, referred to as electronic dispersion compensation (EDC) [3].

An EDC receiver has a better performance for low input powers [3], since in this case the channel is predominantly linear. However, when the input power is high, the Kerr effect becomes significant. In this scenario, to significantly improve the transmission rates, the receiver should also consider the nonlinear effect [4]. To build such a receiver, one may use a mathematical model for the optical channel. These models are generally obtained from the nonlinear Schrödinger equation (NLSE) [1, Ch. 2]. So far in the literature, the solutions of the NLSE are obtained by approximations, which can be numerical or analytical. One of the most accurate approximations is obtained numerically by the split-step Fourier method (SSFM) [5]. In the SSFM, the fiber is represented by a set of small segments, called steps, in which the linear and nonlinear effects can be applied separately. The same method can be applied with the inverse parameters at the receiver and/or transmitter, a process called digital back-propagation (DBP) [6], [7]. DBP can partially compensate the nonlinear and linear effects by choosing a sufficient number of steps. Increasing the number of steps also increases the receiver's computational complexity. To design low-complexity receivers, analytical models are highly desirable.

Analytical models usually consider that either the nonlinear or the linear effect is predominant. In models obtained by degenerated solutions of the NLSE, one of the effects is completely neglected. For example, in the dispersion-only model, the nonlinearity is ignored by setting the nonlinear coefficient $\gamma$ to zero in the NLSE [1, Ch. 3]. In the nonlinear phase noise (NLPN) model, the chromatic dispersion is ignored by setting the group-velocity dispersion (GVD) parameter $\beta_{2}$ to zero [1, Ch. 4], [8]. Analytical models that consider both effects can be obtained by regular perturbation (RP) theory. A well-known model that uses this theory is the $\mathrm{RP}$ on the nonlinear coefficient $\gamma$ [9], [10]. RP on $\gamma$ considers a low nonlinear effect. This model is appropriate for many known communication systems, and has been used for performance prediction and design of receivers [11], [12]. When parameters such as power and $\gamma$ increase, the model loses its accuracy [9]. In that regime, a new model is necessary. Such a model is not available in the literature yet and will be addressed on this paper.

In this paper, we present a novel model based on RP on the GVD parameter $\beta_{2}$ for a lossless NLSE. In contrast to the RP on $\gamma$, the proposed model considers a low dispersion effect and can represent fiber transmission in the highly nonlinear regime. We compare this model with the dispersion-only, NLPN, and RP on $\gamma$ models and analyze their accuracy for different input powers and modulation formats. The proposed model shows excellent performance for input powers greater than $-1 \mathrm{dBm}$ when compared to the other three models. The limits of the model with respect to the bandwidth and fiber length are also analyzed.

\section{OPTICAL CHANNEL MODELS}

In this section, we present four models for the optical channel. The two first models are degenerated solutions of the NLSE. The third is an RP on the nonlinear coefficient $\gamma$ and the fourth is our proposed model, an RP

This work is supported by the Netherlands Organisation for Scientific Research (NWO) via the VIDI Grant ICONIC (project number 15685). The work of A. Alvarado has received funding from the European Research Council (ERC) under the European Union's Horizon 2020 research and innovation programme (grant agreement No 757791). 
on the GVD parameter $\beta_{2}$.

The NLSE for the lossless and noiseless propagation at the retarded frame $t$ and distance $z$ is [1, Ch. 2]

$$
\frac{\partial A(t, z)}{\partial z}=-\frac{j \beta_{2}}{2} \frac{\partial^{2} A(t, z)}{\partial t^{2}}+j \gamma|A(t, z)|^{2} A(t, z),
$$

where $\gamma$ is the nonlinear Kerr coefficient and $\beta_{2}$ is the GVD parameter. We obtain the solution $A$ of (1) numerically using the SSFM. To compare this solution with the signal $A_{M}$ obtained from a model, the normalized square deviation (NSD) metric is used. The NSD is defined as [9, eq. 27]

$$
\mathrm{NSD} \triangleq \frac{\int_{-\infty}^{\infty}\left|A_{M}(t, z)-A(t, z)\right|^{2} \mathrm{~d} t}{\int_{-\infty}^{\infty}|A(t, z)|^{2} \mathrm{~d} t} .
$$

A model will be considered accurate if it has an NSD lower than $0.1 \%$. The power in which a model has that value of NSD is defined as $P_{0.1 \%}$ in this paper.

\subsection{Dispersion-only model}

If we set $\gamma=0$, (1) becomes

$$
\frac{\partial A_{M}(t, z)}{\partial z}=-\frac{j \beta_{2}}{2} \frac{\partial^{2} A_{M}(t, z)}{\partial t^{2}}
$$

which admits a solution, given in the Fourier domain by [1, Ch. 3]

$$
\tilde{A}_{M}(\omega, z)=\tilde{A}(\omega, 0) e^{\frac{j \beta_{2}}{2} \omega^{2} z} \text {. }
$$

We call the solution of (4) the dispersion-only model. This model is an all-pass filter, and causes pulse-width variation [1, Ch. 3], [13, Sec. 3.1.1].

\subsection{Nonlinear phase noise model}

By setting $\beta_{2}=0$, the NLSE in (1) turns into

$$
\frac{\partial A_{M}(t, z)}{\partial z}=j \gamma\left|A_{M}(t, z)\right|^{2} A_{M}(t, z),
$$

which also admits an analytical solution [1, Ch. 4]

$$
A_{M}(t, z)=A(t, 0) e^{j \gamma|A(t, 0)|^{2} z} .
$$

The solution (6) is called the NLPN model. In analogy with the dispersion-only model, the NLPN model does not affect the amplitude of the time-domain signal. In the frequency domain, (6) causes spectral variation [1, Ch. 4], [13, Sec. 3.1.2].

\subsection{Regular Perturbation on $\gamma$}

The first order of a regular perturbation on $\gamma$ is a model that consider the dispersion effect as the main fiber effect. The model is described in the frequency domain as [9]

$$
\tilde{A}_{M}(\omega, z)=\tilde{A}(\omega, 0) e^{\frac{j \beta_{2}}{2} \omega^{2} z}+j \gamma \int_{0}^{z} e^{\frac{j \beta_{2}}{2} \omega^{2}(z-u)} \tilde{S}(\omega, u) \mathrm{d} u,
$$

where $\tilde{S}$ is the Fourier transform of

$$
S(t, z)=\left|A_{0}(t, z)\right|^{2} A_{0}(t, z),
$$

and $A_{0}(t, z)$ is the inverse Fourier transform of $\tilde{A}(w, 0) e^{\frac{j \beta_{2}}{2} w^{2} z}$ in (4). The first term in the right-hand side of (7) accounts only for the dispersion effect. The second term considers the nonlinear and the dispersion effects together. This can be seen by the similarity between the function $S$ and the last term in (1).

2.4 Proposed Model: Regular Perturbation on $\beta_{2}$

We present a first-order RP on $\beta_{2}$. In this model, the main effect is nonlinearity. The model is given by

$$
A_{M}(t, z)=A(t, 0) e^{+j \gamma|A(t, 0)|^{2} z}+\beta_{2} B(t, z) e^{+j \gamma|A(t, 0)|^{2} z}
$$

where

$$
\begin{aligned}
& B(t, z)=-\int_{0}^{z} F(t, u) \mathrm{d} u-2 j \gamma A(t, 0) \Re\left\{A^{*}(t, 0) \int_{0}^{z}(z-u) F(t, u) \mathrm{d} u\right\}, \\
& F(t, z)=\frac{j}{2} \frac{\partial^{2}}{\partial t^{2}}\left\{A(t, 0) e^{+j \gamma|A(t, 0)|^{2} z}\right\} e^{-j \gamma|A(t, 0)|^{2} z} .
\end{aligned}
$$


The first term in the right-hand side of (9) accounts for the nonlinear effect only. The second term also accounts for the mix of dispersion and nonlinear effects. The relationship between this last term and the dispersion is observed in the second-order derivative in (11). In this paper, we motivate the model by numerical simulations, deferring the theoretic analysis to a future publication due to space constrains.

\section{NUMERICAL ANALYSIS}

\subsection{System Parameters}

In the system under consideration, we transmit symbols using $M$-ary quadrature amplitude modulation ( $M$ QAM). The value of $M$ is varied to analyze the impact of the modulation format on the models. For $M=4$, the 4-QAM modulation is called quadrature phase-shift keying (QPSK). The symbols modulate the amplitude of root-raised cosine (RRC) pulses with a roll-off factor of 0.1 . The resulting signal is transmitted over a nonzero dispersion-shifted fiber (NZDSF) with parameters given in Table I.

\begin{tabular}{|c|c|} 
TABLE I: Fiber parameters \\
\hline Parameter & Value \\
\hline Wavelength $\lambda[\mathrm{nm}]$ & 1550 \\
Symbol Rate $[\mathrm{Gbaud}]$ & 10 \\
Fiber Length $[\mathrm{km}]$ & 60 \\
$\beta_{2}\left[\mathrm{ps}^{2} / \mathrm{km}\right]$ & -5.42 \\
$\gamma[1 / \mathrm{W} / \mathrm{km}]$ & 1.46 \\
\hline
\end{tabular}

In an NZDSF, the value of $\left|\beta_{2}\right|$ is lower than in a standard single-mode fiber (with typically $\beta_{2}=-21.67$ $\mathrm{ps}^{2} / \mathrm{km}$ ). This fact provides a regime where the accumulated dispersion is low for the respective fiber length and symbol rate. Changing these two last parameters significantly affect the model accuracy. This will be investigated in the next section.

\subsection{Simulation Results}

Fig. 1(a) shows the NSD in (2) versus input power for the four models with QPSK modulation. As depicted in Fig. 1(a), the dispersion-only and NLPN models have a worse performance than the RP on $\beta_{2}$ in the entire simulated range of powers. While for RP on $\beta_{2} P_{0.1 \%} \approx 8.7 \mathrm{dBm}$, the second highest $P_{0.1 \%}$ is approximately $2.4 \mathrm{dBm}$, for RP on $\gamma$. This difference shows that the RP on $\beta_{2}$ is accurate until powers more than $6.3 \mathrm{~dB}$ greater than the other models. The higher $P_{0.1 \%}$ of RP on $\beta_{2}$ shows more tolerance to power changes, even though $\mathrm{RP}$ on $\gamma$ is more accurate for powers below $-2 \mathrm{dBm}$. Fig. 1(a) also illustrates the dispersion-only model with rectangular 8-QAM modulation in a reduced range of powers. The change in the modulation format introduces a difference $\Delta$ between $P_{0.1 \%}$ for 8-QAM and QPSK of $-1.57 \mathrm{~dB}$.

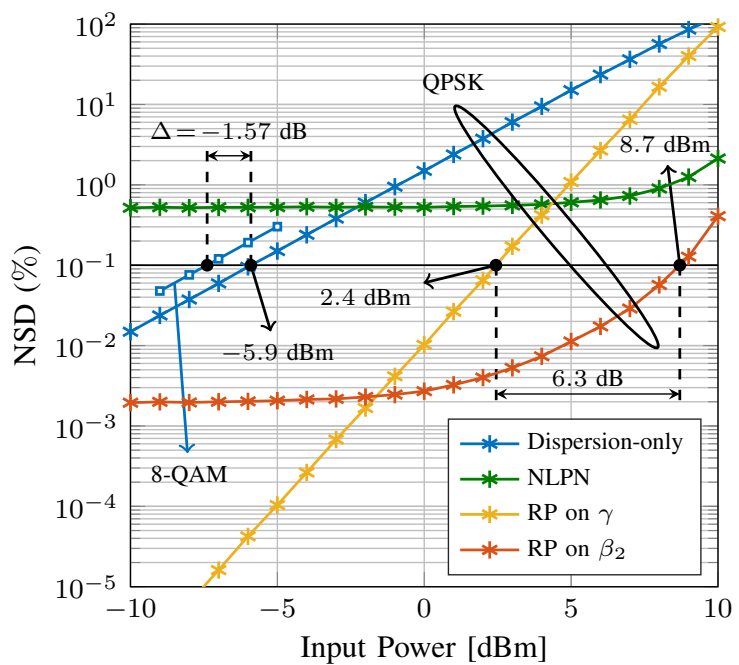

(a) QPSK.

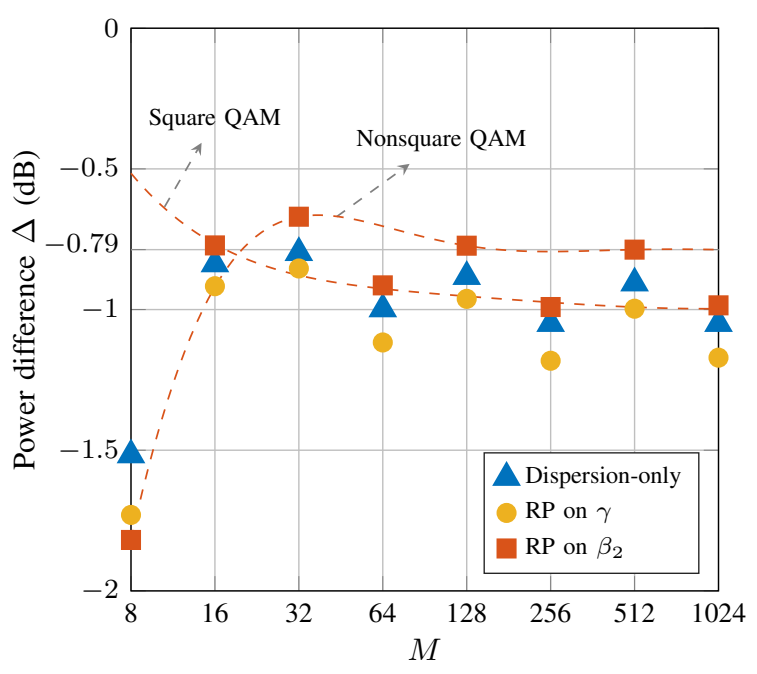

(b) $P_{0.1 \%} \%$ difference with respect to QPSK.

Figure 1: Simulation results for the system given by the parameters in Table I. (a) NSD versus input power for the four different model with QPSK modulation. (b) Difference $\Delta$ between $P_{0.1} \%$ for $M$-QAM modulation $(M \geq 8)$ and QPSK.

The difference $\Delta$ is generalized in Fig. 1(b) for other modulation formats, always with respect to QPSK. Fig. 1(b) illustrates the difference $\Delta$ between $M$-QAM modulation formats and QPSK for the dispersion-only, $\mathrm{RP}$ on $\gamma$ and RP on $\beta_{2}$ models. The NLPN is not considered in this figure since it does not cross the $0.1 \%$ 
threshold (see Fig. 1(a)). As shown in Fig. 1(b), the three models have a negative $\Delta$ for $M \geq 8$. For $M \geq 16$, RP on $\gamma$ has a lower $\Delta$ than the other models. The lowest $\Delta$ for the three models occurs at $M=8$, which is the most nonsquare $M$-QAM. For that modulation format, RP on $\beta_{2}$ presents a $P_{0.1 \%} 1.82 \mathrm{~dB}$ lower than for QPSK. Nevertheless, the other nonsquare modulation formats (odd $\log _{2} M$ ) converge to a higher $\Delta$ than the square ones (even $\log _{2} M$ ). This behavior is depicted with dashed lines for RP on $\beta_{2}$, with $\Delta=-0.79 \mathrm{~dB}$ and $\Delta=-1.00 \mathrm{~dB}$, respectively.

Fig. 2 shows the NSD for RP on $\beta_{2}$ at different combinations of symbol rate and fiber length, considering QPSK. As illustrated in Fig. 2, increasing the fiber length and/or the symbol rate deteriorates the performance of the model. This behavior can be justified using (4). The dispersion effect increases with the distance and square of the signal bandwidth, and the later increases linearly with the symbol rate. Therefore, the approximation made by $\mathrm{RP}$ on $\beta_{2}$ becomes worse, increasing the NSD. Using the same symbol rate of 10 Gbaud used in the previous example, the fiber could be extended to approximately $87.4 \mathrm{~km}$ keeping the model accurate ( $\Delta$ in Fig. 2 ). Fixing the fiber length to $60 \mathrm{~km}$, the symbol rate could be increase to only 13.4 Gbaud ( $\square$ ). When targeting higher symbol rates, the reach distance is significantly decreased. For 30 Gbaud, the model is accurate for a maximum fiber length of $17.0 \mathrm{~km}(\otimes)$.

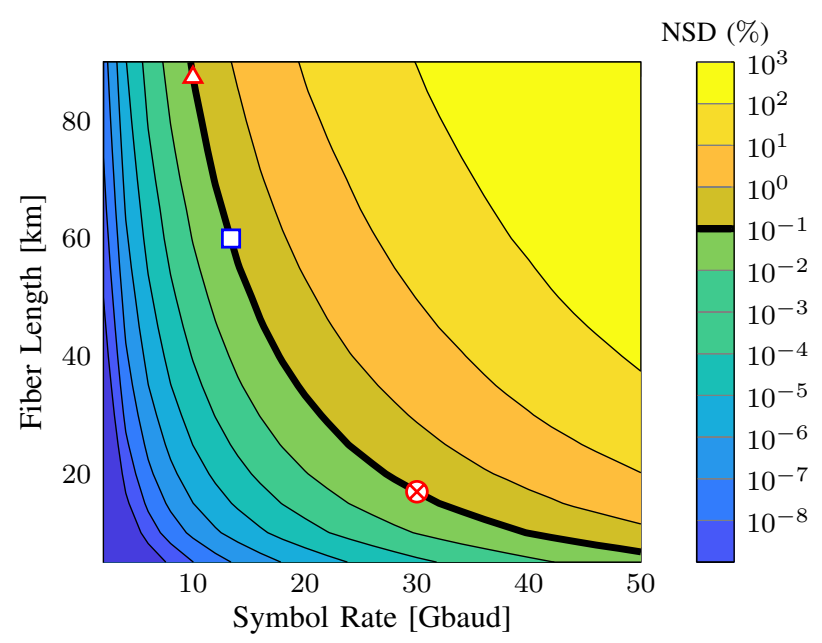

Figure 2: NSD for RP on $\beta_{2}$ at different symbol rates and fiber lengths. We considered a QPSK signal with $5 \mathrm{dBm}$ input power.

\section{CONCLUSIONS}

We presented in this paper a novel model for the weak-dispersion regime in a lossless fiber, which can be used even with high nonlinearity. The proposed model accurately represents the fiber output until an input power of $8.7 \mathrm{dBm}$ in the system under consideration. The modulation format impacts the performance of the models, especially when comparing QPSK and 8-QAM. The effect of changes in the symbol rate and fiber length was also analysed, which showed a high sensitivity of the model to these two parameters. Deriving a receiver based on the proposed model and finding higher-order perturbations on $\beta_{2}$ are possible extensions of the work.

\section{ACKNOWLEDGEMENTS}

The authors would like to thank Dr. Tobias Fehenberger, Dr. Nicola Calabretta, Dr. Domaniç Lavery and Dr. Gabriele Liga for fruitful discussions.

\section{REFERENCES}

[1] G. Agrawal: Nonlinear Fiber Optics, Academic Press, Boston, 2013, 5th edn.

[2] R.-J. Essiambre et al.: "Capacity limits of optical fiber networks," J. Lightw. Technol., 28 (4), 662-701, 2010.

[3] H. Blow, F. Buchali, and A. Klekamp: "Electronic dispersion compensation,” J. Lightw. Technol., 26 (1), 662-701, 2010.

[4] S. T. Le, V. Aref and H. Buelow: "Nonlinear signal multiplexing for communication beyond the Kerr nonlinearity limit," Nat. Photonics 11, 570-577, 2017.

[5] H. Blow, F. Buchali, and A. Klekamp: "Optimization of the split-step Fourier method in modeling opticalfiber communications systems," J. Lightw. Technol., 21 (1), 61-68, 2003.

[6] R.-J. Essiambre and P. J. Winzer: "Fibre nonlinearities in electronically pre-distorted transmission," in Proc. ECOC, Glasgow, United Kingdom, 2005, paper Tu 3.2.2.

[7] E. Ip and J. M. Kahn: "Compensation of dispersion and nonlinear impairments using digital backpropagation,” J. Lightw. Technol., 26 (20), 3416-3425, 2008.

[8] L. Beygi, E. Agrell, M. Karlsson, and P. Johannisson: "Signal statistics in fiber-optical channels with polarization multiplexing and self-phase modulation," J. Lightw. Technol., 29 (16), 2379-2386, 2011.

[9] A. Vannucci, P. Serena, and A. Bononi: "The RP method: a new tool for the iterative solution of the nonlinear Schrödinger equation," J. Lightw. Technol., 20 (7), 1102-1112, 2002.

[10] E. Forestieri, and M. Secondini: "Solving the nonlinear Schrödinger equation," in Optical Communication Theory and Techniques, E. Forestieri, Springer US, Boston, 3-11, 2011.

[11] W. Yan et al.: "Low complexity digital perturbation backpropagation," in Proc. ECOC, Geneva, Switzerland, 2011, paper Tu.3.A.2.

[12] P. Poggiolini et al:: "The GN-model of fiber non-linear propagation and its applications," J. Lightw. Technol., 32 (4), 694-721, 2014.

[13] J. K. Shaw: Mathematical Principles of Optical Fiber Communications, Society for Industrial and Applied Mathematics, Philadelphia, 2004. 